\title{
Analysis of Switching Overvoltage in Regional Grid with Small Hydropower Plants
}

\author{
Juan Chen, Haifeng Li \\ Electric Power College, South China University of Technology, Guangzhou, China \\ Email: 362360029@qq.com \\ Received June 2013
}

\begin{abstract}
Present-day small hydropower plants (SHPs) have a large development potential because of the increasing interest in renewable resources and distributed energy generation, therefore, there are many SHPs in places of China where are rich in water resources. However, it has caused overvoltages in the distribution network, and which is even worse for the switching overvoltage such as isolated network operation, changing power supply path. The simple network model is used to analyze the reasons of the switching overvoltage, and the simulation software DIgSILENT/PowerFactory is used to check out the results of the theoretical analysis.
\end{abstract}

Keywords: Small Hydropower; Isolated Operation; Changing Power Supply Path; Switching Overvoltage

\section{Introduction}

Hydropower is an important part of global energy production with a rapid growth in recent years. Therefore, there is a strong demand for the development of SHP (short for small hydropower) for making the most of clean hydropower. Ended to 2005, there are 50,000 SHPs, widely distributed in more than 30 provinces in China, the capacity of which is up to 38,530 MW [1]. The SHPs are constructed in remote mountainous regions, besides, they are primarily runoff hydropower plants defined as providing little or no water storage, of which the active power is nonadjustable, and hard to control $[2,3]$.

Because of the long power transmission distance, the small diameter of the transmission line, and the suppression of the grid voltage, overvoltage and high power factor are common problems of the SHPs [4]. What we should pay more attention to is, when the primary power supply line is cut off, the SHPs still work as an isolated network with the local load. If the power of the SHPs is more than that of the loads, the isolated network will suffer overvoltage and high frequency with damages to the electrical equipment. Additionally, when the primary power supply line requires maintenance, the SHPs should change to another power supply path to prevent the loads' power from being cut off, in case that the power supply path is changed to a longer one (only this kind of changing power supply path is discussed this paper), which will lead to overvoltage as well.

Currently, researches of SHP mainly focus on two parts, the one is the reasons for overvoltage and high power factor, including large transmission losses, nonadjustable step-up transformer, and fierce conflicts between the dry season and the wet season [5]; the other is the assessment of SHP's reactive power based on optimal power flow (OPF) [6]. Although the overvoltage problem of SHP has been involved, there's no rigorous theoretical derivation for the switching overvoltage especially in isolated operation and changing power supply path.

Therefore, in this paper, theoretical analyses are made for the two kinds of switching overvoltage by the simple network model; and the results of theoretical analysis are verified by the simulation software DIgSILENT/PowerFactory, meanwhile, some effectual advices are proposed to restrain the switching overvoltage.

\section{Analysis of Overvoltage in Region Grid with Small Hydropower}

The simple network model given in Figure 1 consists a synchronous generator, a step-up transformer, the transmission line, a load and the external network. The synchronous generator runs as an isolated network with the local load when the breaker connected to the external network and previously closed is open. Without automatic excitation regulator, the SHP can be equivalent to be a constant voltage source with resistance in theoretical analyses.

\subsection{Overvoltage in Isolated Operation}

The regional grid always runs as an isolated network when the primary power supply line is cut off because of 


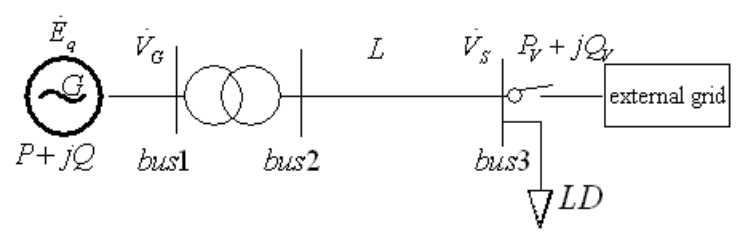

Figure 1. Model of a simple network.

faults or other reasons. The voltage of bus3 shown in (1) can be deduced by the model of the simple network.

$$
V_{S}=\frac{E_{q} \cos \delta+\sqrt{\left(E_{q} \cos \delta\right)^{2}-4 K\left(R_{\Sigma} P_{V}+X_{\Sigma} Q_{V}\right)}}{2 K}
$$

where, $\quad K=1+\left(R_{\Sigma} R_{L D}+X_{\Sigma} X_{L D}\right) /\left(R_{L D}^{2}+X_{L D}^{2}\right), \quad E_{q}$ the no-load voltage of the generator, $\delta$ is the angle between $E_{q}$ and $V_{S}, Z_{\Sigma}$ is the total impedance of the synchronous generator, transmission line and step-up transformer.

From (1), it can be seen clearly that if $P_{V}$ and $Q_{V}$ reduce to 0 , the voltage of bus 3 will rise sharply. Actually, the SHPs supply power to both the local loads and the external network (means $P_{V}>0, Q_{V}>0$ ) in wet season, when line fault (leading to the isolated operation) occurs frequently by the thunderstorm. Thevenin's theorem can be used in the complex electricity network, so that the complex network can be equivalent to a constant voltage source with resistance, which is the same as the simple network, so does the analysis method and the conclusion.

\subsection{Overvoltage in Changing Power Supply Path}

To ensure power distribution reliability, the SHPs change to another power supply path when the primary power supply line requires maintenance. Figure 1 shows that the voltage of bus connected to the external grid is $V_{S}$, the power at $E_{q}$ is $P+j Q$ :

$$
\begin{aligned}
& P=\frac{R_{\Sigma} E_{q}{ }^{2}-Z_{\Sigma} E_{q} V_{S} \cos \left(\varphi_{\Sigma}+\delta\right)}{Z_{\Sigma}{ }^{2}} \\
& Q=\frac{X_{\Sigma} E_{q}{ }^{2}-Z_{\Sigma} E_{q} V_{S} \sin \left(\varphi_{\Sigma}+\delta\right)}{Z_{\Sigma}{ }^{2}}
\end{aligned}
$$

where $\varphi_{\Sigma}$ is the impedance angle of $Z_{\Sigma}$.

The voltage $V_{S}$ keeps invariable for the suppression of the external grid, total impedance $Z_{\Sigma}$ increases. From (2) we can know that the electromagnetic power of SHP will reduce, while the mechanical power holds changeless because the water inflow keeps the same, thus, the electromagnetic power is less than the mechanical power, so that the rotor speeds up, and the power angle rises up.

The resistance is almost equal to the reactance in distribution network, so we can't ignore the resistance while making theoretical analysis. In addition, more than $90 \%$ of $Z_{\Sigma}$ is the transmission line impedance, so the power angle is about $50^{\circ}$. Both the increase of the power angle $\delta$ and the total impedance $Z_{\Sigma}$ will decrease the reactive power (see (3)).

At this stage, the power management department take power factor as assessment for the SHPs, if the SHP generates less reactive power than that required, each $3 \mathrm{Kv}$ rah is equal to the benefit of $1 \mathrm{kWh}$ active power as a punishment. Based on the analysis above, the power factor gets higher and can't meet the assessment requirement in changing power supply path, as a consequence, the excitation voltage of the SHPs are raised up.

The reactive power against terminal voltage of SHP can be expressed as (4) according to (3):

$$
Q=\frac{X_{S} E_{q}^{2}-Z_{S} E_{q} V_{G} \sin \left(\varphi_{S}+\delta^{\prime}\right)}{Z_{S}^{2}}
$$

meanwhile

$$
V_{G}=\frac{X_{S} E_{q}^{2}-Z_{S}^{2} Q}{Z_{S} E_{q} \sin \left(\varphi_{S}+\delta^{\prime}\right)}
$$

where $X_{S}$ is the internal reactance of generator, $\varphi_{S}$ is the impedance angle of $Z_{S}, \delta^{\prime}$ is the angle between $\dot{E}_{q}$ and $\dot{V}_{G}$.

The impedance angle of $Z_{S}$ is close to $90^{\circ}$ because of the small internal impedance of the generator. From (5) we know that both the increase of $\delta^{\prime}$ and $Q$ lead to the increase of $V_{G}$, similarly, all the bus voltage shown in Figure 1 will increase, then the whole network suffers from overvoltage while the SHPs' excitation voltage is raised up at the same time.

\section{Case Study}

The Shaoguan grid is rich in hydropower resources, where the installed capacity of hydropower has reached $1700 \mathrm{MW}$ at the end of 2005. We select Bibei town Ruyuan district Shaoguan as the overvoltage verification point, because the available water resources reserves amount to $104,500 \mathrm{~kW}$ (theoretical potential), the actual available water is up to $76,000 \mathrm{~kW}$ and currently there has developed two medium-sized hydropower stations (Hengxi plant) and more than 10 SHPs. Figure 2 shows the distribution network of $10 \mathrm{kV}$ F36 Bibei line of Hengxi plant and $10 \mathrm{kV}$ Daqiao line of Daqiao substation, and it has been built for simulation experiments in DIgSILENT/PowerFactory. Overvoltages are defined in perunit values (p.u.).

\subsection{Overvoltage in Isolated Operation}

We set a short circuit on the line between Daling and Wangcha at $30 \mathrm{~s}$, and the breakers at each end of the line (without the function of automatic reclosing) opens, that makes the areas including Wangcha, Nakeng, Qingshi, 


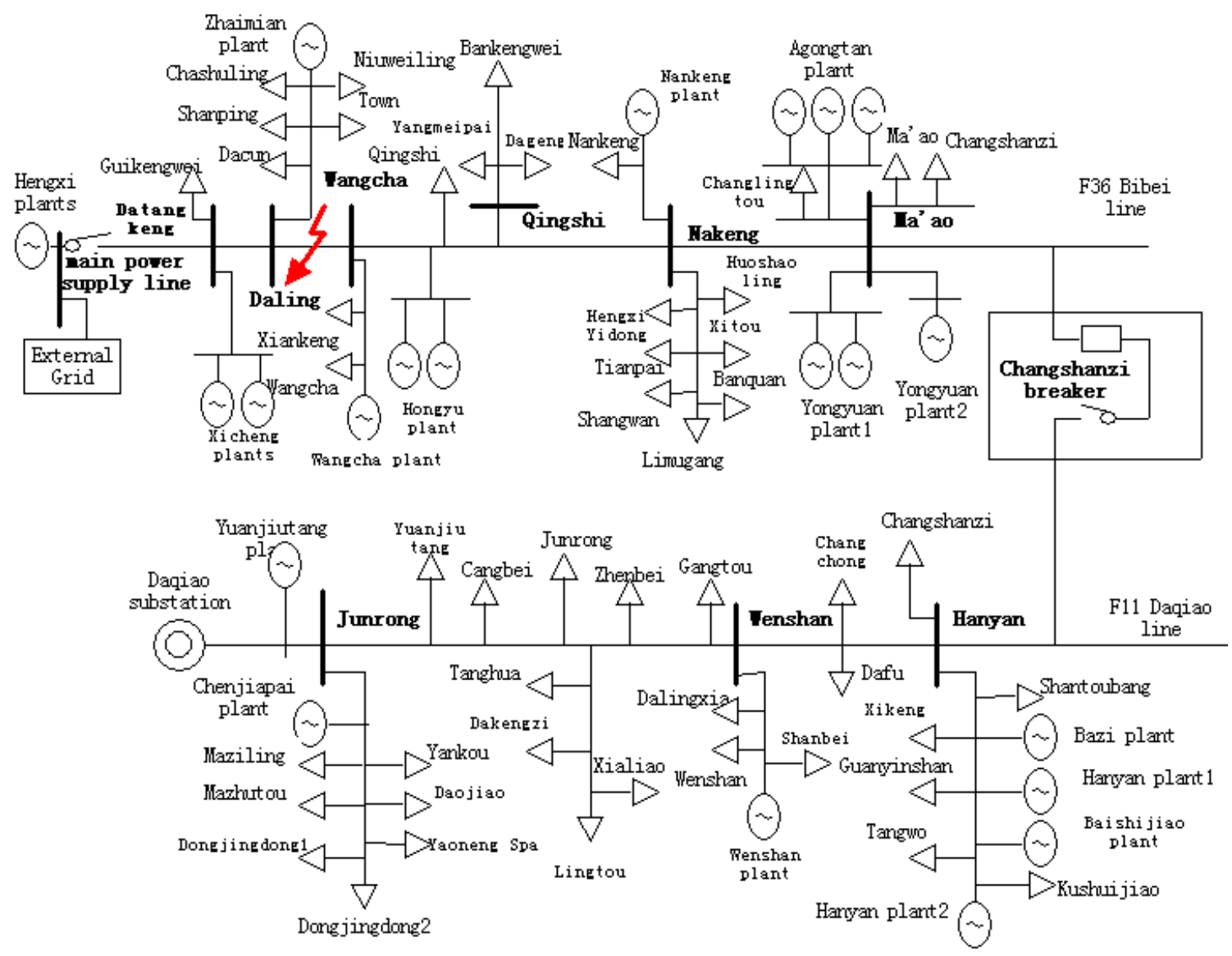

Figure 2. Distribution network of $10 \mathrm{kV}$ F36 Bibei line of Hengxi plant and $10 \mathrm{kV}$ Daqiao line of Daqiao substation.

Ma'ao operate as an isolated network with the 10 SHPs in 5 hydropower plants, such as Wangcha plant, Hongyu plant, Nankeng plant, Yongyuan plant and Agongtan plant. The bus voltage of Wangcha and Nankeng are shown in Figure 3:

We can see it clearly that the buses' voltage are all increased, the bus voltage of Wangcha rises to 2.342 p.u. from 1.056 p.u., while the bus voltage of Nankeng is up to 2.340 p.u. from 1.066 p.u. Thus, the isolated operation results in serious overvoltage.

\subsection{Overvoltage in Changing Power Supply Path}

The contacted switch named Changshanzi changes the state from cold standby to hot standby, as a result, the power of Bibei Town is supplied by Daqiao substation instead of Hengxi plant. We disconnect the primary power supply line by opening the outlet circuit breaker of Hengxi plant at $t=30 \mathrm{~s}$, and close the Changshanzi breaker at $\mathrm{t}=32 \mathrm{~s}$ to simulate the process of changing power supply path.

It is obvious in Figure $\mathbf{4}$ and Figure $\mathbf{5}$ that the bus voltage of Daling reaches the value of 1.21 p.u. from 1.037 p.u., and the bus voltage of Wangcha is up to 1.202

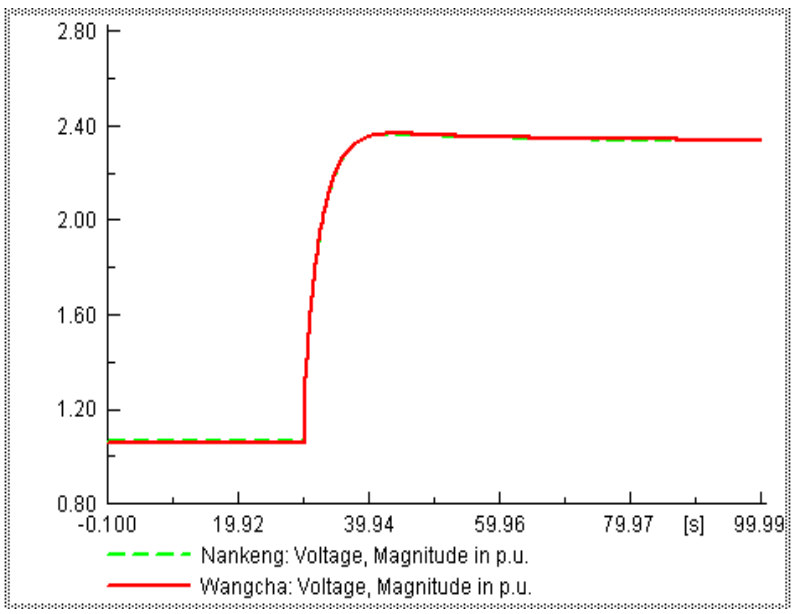

Figure 3. Bus voltage of Datangkeng and Guikeng in isolated operation.

p.u. from 1.034 p.u. On the other hand, the reactive power of Zhaimian plant is reduced from 0.105 MVar to 0.069 MVar, the power factor is over the assessment scope by rising from 0.925 to 0.966 , which makes the excitation voltage raised and the whole network overvoltaged. 


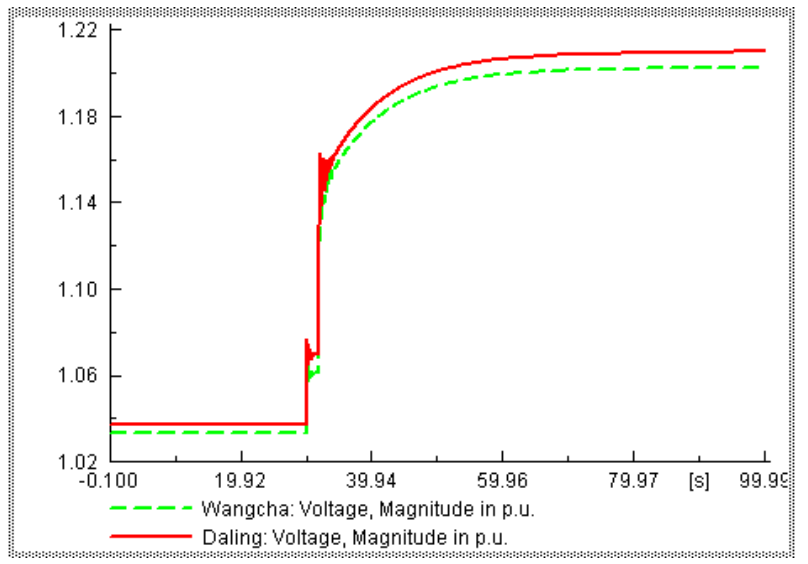

Figure 4. Bus voltage of Daling and Wangcha in changing power supply path.

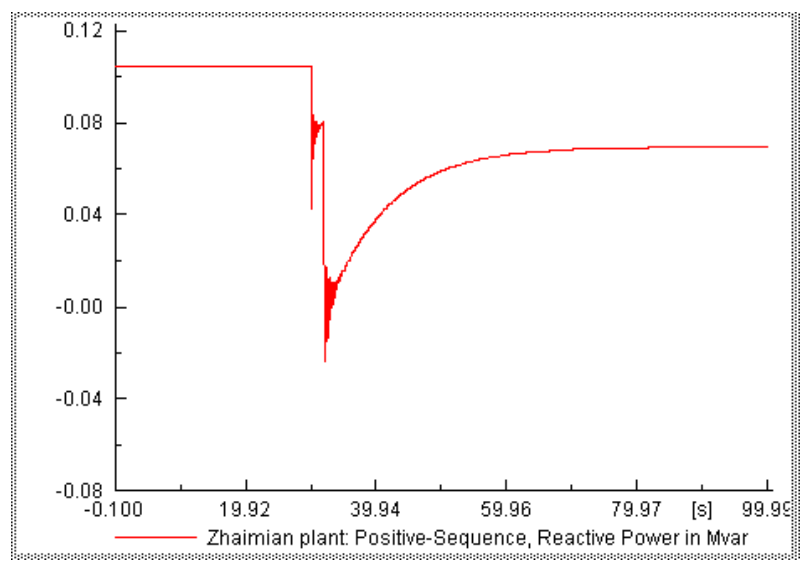

Figure 5. The reactive power of Zhaimian plant in changing power supply path.

\section{Some Effective Measures to Restrain Switching Overvoltage in Region Grid}

There are many measures to restrain overvoltage, the following three must be more effective.

1) Put into intelligent automatic bus transfer equipment. The quality of voltage and frequency in the isolated network are too poor which can often damage the electrical appliances. The conventional automatic bus transfer equipment has the defect of needing more time to detect bus non-voltage, at the cost of islanding all the SHPs and taking a very long time to restore power supply. In this case, not only the power quality is poor, but also the reliability of power supply is low. The automatic bus transfer has two modes, the one is that the SHPs are quickly selected and tripped in low water period, while the other one (in the mode of high frequency) is that the SHPs are tripped round by round and the automatic synchronous capture reclose in high flow period, which enables regional power supply to restore more quickly and has greatly improved the quality of frequency and voltage [7].
2) Equip the small hydropower with automatic excitation regulator. As we all know that the AVR (short for automatic excitation regulator) can regulate the voltage automatically, better distribution of the system's reactive power. It is clear from formulas (2) and (3) that $E_{q}$ has a significant effect on the active power and reactive power of the generators. The unbalanced power which can cause great disturbance to the system and is not conducive to the stability of the system can be reduced by the regulation of the AVR. Putting into the AVR for SHPs has significant effect on restraining overvoltages.

Install the voltage control terminal of smart distribution grid in the load side. The voltage control terminal makes use of the modern power electronic technology, and its primary components include silicon controlled rectifier and the PWM inverter, which can effectively achieve the isolation and control of the voltage. The terminal can convert the unqualified voltage into the one that become available to be used by the user directly, so as to keep the overvoltages from damaging the electrical equipment effectively.

\section{Conclusions}

On the basis of the analysis of theory and simulation in overvoltages, the following conclusions can be drawn:

1) There will be severe overvoltages in isolated operation, changing power supply path and stepping into the wet season.

2) Some effective measures have been proposed to restrain overvoltage in region grid, like putting into intelligent automatic bus transfer equipment in order to restore power supply more quickly; equipping the SHPs with AVR to regulate the too high or too low voltage automatically; and installing the voltage control terminal in the load side to prevent the overvoltages from damaging the electric equipment.

\section{Acknowledgements}

The work described in this paper is supported by Guangdong Special Fund Project of Industry, University and Research Institute Collaboration (2011A090200127, 2011A090200074).

\section{REFERENCES}

[1] X. Chen, "Technology Development of the Small Hydropower Resources in China," Rural Electrification, Vol. 1, 2006, pp. 40-42.

[2] T. F. Zhou and Z. Y. Wang, "The Problems and Countermeasures of Small Hydropower Development and Construction in China," China Rural Water and Hydropower, Vol. 2, 2007, pp, 82-84.

[3] F. H. Jiang and X. Z. Du, "Development Status and Problems of Small Hydropower in China,” China Rural 
Water and Hydropower, Vol. 3, 2004, pp. 82-83.

[4] H. L. Zhang and L. G. Liu, "Voltage and Reactive Power of Small Hydropower System and Study on Control Strategy," Master Thesis, North China Electric Power University, Beijing, 2010.

[5] X. H. Fang, "Management of Voltage and Reactive Power of Small Hydropower," Rural Electrification, Vol. 7, 2008.

[6] L. QIAO, L. Guo and R. Zhang, "Estimated Means of
Reactive Power for Distributed Small Hydropower Based on Optimal Power Flow," Proceedings of the Chinese Society of Universities, Vol. 2, 2008.

[7] Z. F. Chen, Y. Wu and D. Q. Jin, "Research on Intelligent Automatic Bus Transfer Equipment Suitable for the Small Hydroelectric Power System in Mountainous Area," Power System Protection and Control, Vol. 38. No. 8, 2010, pp. 120-122. 